\title{
Output Correlation and EMU: Evidence from European Countries
}

\author{
Kazuyuki Inagaki \\ Graduate School of Economics, Kobe University
}

\begin{abstract}
This paper examines the endogenous nature of business cycle synchronization. Using the residual cross-correlation approach, we explore output correlation between European EMU member countries. Our findings can be summarized as follows: (i) contemporaneous correlation estimates become more significant during the EMU period; (ii) business cycles are dependent but not highly synchronized during the pre-EMU period; (iii) after the transition to EMU, business cycles are dependent and more highly synchronized. These empirical results suggest that costs arising from asymmetric business cycles have a tendency to decrease. On these grounds, we conclude that the endogeneity of OCA hypothesis holds in the case of the euro area.
\end{abstract}

- JEL classification: E32, F15

- Keywords: EMU, Endogeneity of OCA, Output correlation

\section{Introduction}

International output correlation is a useful measure of business cycle synchronization across countries. A number of studies have been made on international output correlation, especially in examining the process of Economic and Monetary Union (EMU). Using a common currency, an EMU member country can reinforce trade and financial integration with other EMU member countries. The start of EMU is likely to induce a considerable change in the degree of

\footnotetext{
*Corresponding address: Kazuyuki, Inagaki, Graduate School of Economics, Kobe University, Rokkodai, Nada-ku, Kobe 657-8501, Japan. Tel: , Fax: E-mail: 027d252@stu.kobe-u.ac.jp (C2006-Center for International Economics, Sejong Institution, All Rights Reserved.
} 
business cycle synchronization across EMU member countries. There is general agreement on this point (e.g., Artis and Zhang, 1995, 1999; Frankel and Rose, 1998, 2002; Kalemli-Ozcan et al., 2003; Krugman, 1991; Mélitz 2004; Ramos et al. 2003; Rose and Engle, 2001; see also De Grauwe, 2005, for a detailed survey).

However, economic theory does not provide definitive guidance concerning the impact of EMU on the degree of business cycle synchronization. Expansion of intra-industry trade allows similar demand shocks to easily spread across trading partners; thus, one possibility is to assume that EMU leads to an increase in the degree of business cycle synchronization across EMU member countries (e.g., Frankel and Rose, 1998). At the same time, trade integration may increase countryspecific shocks owing to regional concentration of industrial activities arising from economies of scale; consequently, there is another possibility that EMU gives rise to a decrease in the degree of business cycle synchronization across EMU member countries (e.g., Krugman, 1991). From the theoretical point of view, the endogenous nature of business cycle synchronization is still in controversy. Hence, it is worthwhile examining this subject empirically to elucidate the impact of EMU on the degree of business cycle synchronization.

This paper is intended as an empirical investigation on the endogeneity of optimum currency area (OCA). The endogeneity of OCA hypothesis, as Frankel and Rose (1998) point out, implies that a currency area turns into an OCA ex post through high business cycle synchronization consequent on the formation of EMU. ${ }^{1}$ Accordingly, it follows from the above that there are two directly-opposed ideas concerning the endogeneity of OCA hypothesis: the hypothesis holds in the former case, but not in the latter case. Output series and their correlation patterns are especially useful for understanding the degree of business cycle synchronization across EMU member countries. To characterize output correlation patterns for the EMU period, a comparison with those for the pre-EMU period may be helpful. Therefore, the endogeneity of OCA hypothesis can be tested empirically by checking differences between output correlation patterns for the pre-EMU period and those for the EMU period.

We shall now look briefly at previous empirical studies about the impact of economic integration on international output correlation. Using data for twenty-one industrial countries, Frankel and Rose (1998) find that business cycles are more

'The endogeneity of OCA can originate from several sources other than trade integration. For further details of this point, see De Grauwe (2005). 
highly synchronized for countries with closer trade linkages. This finding is also confirmed in Rose and Engle (2002). Artis and Zhang (1995) examine the impact of Exchange Rate Mechanism (ERM) on the degree of business cycle synchronization and show that the business cycle affiliation of ERM member countries shifts from the United States to Germany owing to the formation of the ERM. Moreover, Ramos et al. (2003) employ data for European EMU member countries and report that the relative importance of country-specific shocks to common sectoral shocks tends to reduce because of intense policy coordination in the euro area. On the contrary, Kalemli-Ozcan et al. (2003) provide empirical evidence that better risk-sharing opportunities arising from capital market integration increase the degree of industrial specialization, and thus the study suggests that financial integration makes international macroeconomic fluctuations less symmetric.

Many previous studies provide empirical evidence that economic integration tends to increase the degree of business cycle synchronization across countries. Hence, candidates for a common currency area are likely to be members of an OCA. From this viewpoint, we may say that the endogeneity of OCA tends to be supported empirically. What seems to be lacking, however, is the detailed analysis of lead-lag relationships (i.e., timing of when output movements in a specific country affect other countries). This analysis provides more information on the degree of business cycle synchronization (e.g., Artis and Zhang, 1995). Although several previous studies pay attention to only contemporaneous correlation, lagged output correlation is an important measure of business cycle synchronization as well. To overlook this point is to miss the nature of business cycle synchronization across EMU member countries.

Standard methods for investigating international output co-movement are regression-based. However, these methods may not provide information on the timing of correlation. In addition, the uncertainty in the potential interaction between output series would complicate the formulation of regression models considerably. Therefore, this paper uses the residual cross-correlation approach to investigate output correlation between EMU member countries. This approach is the test for independence between two jointly covariance-stationary time series. The approach is simple and easier to implement, and provides information on detailed lead-lag relationships. Moreover, the approach is especially useful when researchers expect long lags in correlation patterns. Considering these advantages, we employ this approach in the hope that it will provide new evidence supporting 
the endogeneity of OCA hypothesis.

This paper is organized as follows. The next section describes the empirical methodology. Section 3 details the data. In section 4, we use the residual crosscorrelation approach and investigate output correlation between European EMU member countries. Section 5 concludes this paper.

\section{Methodology}

In this section, we briefly review the residual cross-correlation approach. Haugh (1976) proposed a test for independence between two jointly covariance-stationary time series using the cross-correlation function between two residual series. The cross-correlation function between two original series is a standard tool. However, the application of the usual cross-correlation function may give rise to misleading interpretation on the nature of interaction between two original series. It is well known that the usual cross-correlation estimates between two original series can be difficult to interpret when each of the two original series has the autocorrelation, because the cross-correlation estimates at different lags are also correlated (possibly to a large extent). On the contrary, the residual cross-correlation approach is much easier to handle and interpret because it filters out the autocorrelation of each original series.

Let us look more closely at the residual cross-correlation approach. Suppose that two jointly covariance-stationary time series, $y_{1 t}$ and $y_{2 t}$, are characterized by the following linear processes:

$$
y_{i t}=\sum_{j=0}^{\infty} \theta_{i j} u_{i t-j}=\theta_{i}(L) u_{i t}, \quad i=1,2
$$

where $u_{1 t}$ and $u_{2 t}$ are two independent white noise series with zero mean and finite variance, $\theta_{i 0}=1$, and $L$ is a lag operator. Although both $u_{1 t}$ and $u_{2 t}$ are unobservable, we can use their estimators, $\hat{u}_{1 t}$ and $\hat{u}_{2 t}$, to test the hypothesis.

The Haugh (1976) test is based on the sample cross-correlation function between two residuals obtained from univariate models, respectively. Suppose we have a sample of size $T$. Let $\hat{\rho}_{u_{1} u_{2}}(k)$ be the sample residual cross-correlation function given by 


$$
\hat{\rho}_{u_{1} u_{2}}(k)=\hat{C}_{u_{1} u_{2}}(k)\left\{\hat{C}_{u_{1} u_{1}}(0) \hat{C}_{u_{2} u_{2}}(0)\right\}^{-1 / 2},
$$

where $\hat{C}_{u_{1} u_{2}}(k)$ is the sample cross-covariance function defined as

$$
\hat{C}_{u_{1} u_{2}}(k)= \begin{cases}T^{-1} \sum_{t=1+k}^{T} \hat{u}_{1 t} \hat{u}_{2 t-k}, & k \geq 0, \\ T^{-1} \sum_{t=1-k}^{T} \hat{u}_{2 t} \hat{u}_{1 t+k}, & k<0,\end{cases}
$$

and $\hat{C}_{u_{i} u_{i}}(0)=T^{-1} \sum_{t=1}^{T} \hat{u}_{i t}^{2}$. Under the assumptions and some regularity conditions, $\sqrt{T} \hat{\rho}_{u_{1} u_{2}}(k)$ has an asymptotic normal distribution as follows:

$$
\sqrt{T}\left(\hat{\rho}_{u_{1} u_{2}}\left(k_{1}\right), \ldots, \hat{\rho}_{u_{1} u_{2}}\left(k_{m}\right)\right) \stackrel{L}{\rightarrow} N\left(0, I_{m}\right)
$$

where $k_{1}, \ldots, k_{m}$ are $m$ different integers, $I_{m}$ is the $m \times m$ identity matrix, and $\stackrel{L}{\rightarrow}$ shows the convergence in distribution.

The important point to note is that the residual cross-correlation estimates at different lags can be judged individually against the approximate standard deviation $T^{-1 / 2}$. Under the assumption that two jointly covariance-stationary time series are characterized by the linear processes (1), Haugh (1976) shows that two original series are uncorrelated if and only if two corresponding white noise residual series are uncorrelated. It is quite easy to interpret correlation patterns in this framework because the autocorrelation of each original series is filtered out. The positive lag $(k>0)$ refers to the number of periods that original series 1 lags behind original series 2 , whereas the negative lag $(k<0)$ refers to the number of periods that original series 1 leads original series 2 . Consequently, we can examine detailed lead-lag relationships between two original series by testing the significance of $\hat{\rho}_{u_{1} u_{2}}(k)$ for $k=0, \pm 1, \pm 2, \ldots, \pm m$; and the null hypothesis of independence between two original series is rejected if $\hat{\rho}_{u_{1} u_{2}}(k) \neq 0$ for some $k$.

\section{Data}

The data consists of monthly observations of the seasonally adjusted industrial production index for ten European countries: Austria (AT), Belgium (BE), Finland 
(FI), France (FR), Germany (DE), Ireland (IE), Italy (IT), Portugal (PT), Spain (ES), and the Netherlands (NL). The source is the International Financial Statistics. ${ }^{2}$ The industrial production index is the most representative data to measure output movements on a monthly basis, and offers more observations than GDP. Moreover, industrial production is very sensitive to business cycles, and thus is likely to reflect such phenomena. The index is logged and differenced to archive stationarity. Hence, this paper uses the growth rate of the industrial production index calculated as $y_{t}=100 \times\left(\ln Y_{t}-\ln Y_{t-1}\right)$, where $Y_{t}$ is the industrial production index at time $t$. The growth rates are denoted as $\mathrm{AT}_{t}, \mathrm{BE}_{t}, \mathrm{FI}_{t}, \mathrm{FR}_{t}, \mathrm{DE}_{t}$, $\mathrm{IE}_{t}, \mathrm{IT}_{t}, \mathrm{PT}_{t}, \mathrm{ES}_{t}$, and $\mathrm{NL}_{t}$, respectively.

To characterize correlation patterns for the EMU period, we consider two sample periods: January 1994 to December 1998, and January 1999 to December 2003. This paper uses data from January 1994 in order to exclude the impact of the currency crisis in Europe. Table 1 summarizes results of unit root tests for the growth rate of the industrial production index. The augmented Dickey and Fuller

Table 1. Augmented Dickey-Fuller test and Phillips-Perron test

\begin{tabular}{|c|c|c|c|c|c|c|c|c|c|c|}
\hline & $\mathrm{AT}_{t}$ & $\mathrm{BE}_{t}$ & $\mathrm{DE}_{t}$ & $\mathrm{ES}_{t}$ & $\mathrm{FI}_{t}$ & $\mathrm{FR}_{t}$ & $\mathrm{IE}_{t}$ & $\mathrm{IT}_{t}$ & $\mathrm{NL}_{t}$ & $\mathrm{PT}_{t}$ \\
\hline \multicolumn{11}{|c|}{ January 1994 to December 1998} \\
\hline \multicolumn{11}{|c|}{ Augmented Dickey-Fuller test } \\
\hline $\mathrm{CT}$ & $-6.79 *$ & $-9.72 *$ & $-7.73 *$ & $-10.57^{*}$ & $-7.81 *$ & $-12.13 *$ & $-5.69 *$ & -3.35 & $-9.25^{*}$ & $-9.78 *$ \\
\hline $\mathrm{C}$ & $-9.30^{*}$ & $-9.80 *$ & $-7.80 *$ & $-7.05^{*}$ & $-7.88 *$ & $-12.32 *$ & $-5.76^{*}$ & $-2.94 *$ & $-9.25 *$ & $-9.84 *$ \\
\hline \multicolumn{11}{|c|}{ Phillips-Perron test } \\
\hline CT & $-15.75^{*}$ & $-15.82 *$ & $-12.02 *$ & $-10.90 *$ & $-14.10^{*}$ & $-12.19 *$ & $-12.60 *$ & $-7.01 *$ & $-16.27 *$ & $-17.04 *$ \\
\hline $\mathrm{C}$ & $-15.81 *$ & $-15.96^{*}$ & $-12.14 *$ & $-11.02 *$ & $-14.24 *$ & $-12.26^{*}$ & $-12.73 *$ & $-6.54 *$ & $-16.39 *$ & $-17.03 *$ \\
\hline
\end{tabular}

January 1999 to December 2003

Augmented Dickey-Fuller test

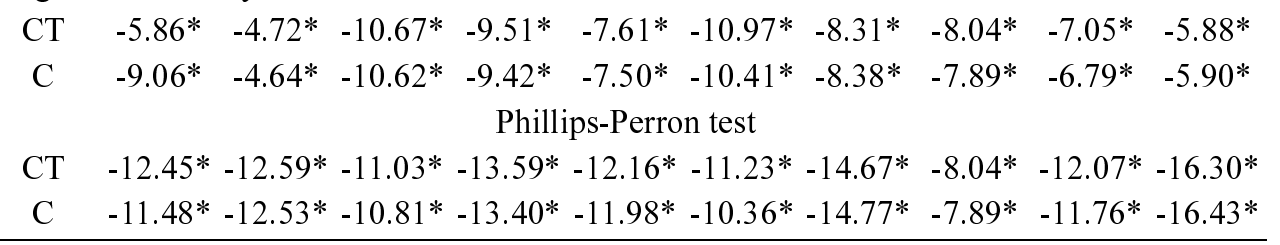

Table 1 indicates results of unit root tests for each differenced series. CT corresponds to the regression including a constant term and a liner trend, and $\mathrm{C}$ corresponds to the regression including a constant term only. * shows that the null hypothesis of a unit root is rejected at the 5 percent significance level.

\footnotetext{
${ }^{2}$ I made use of the database in the Research Institute for Economics and Business at Kobe University.
} 
(1979) test and the Phillips and Perron (1988) test are applied to unit root tests. ${ }^{3}$ As expected, the null hypothesis of a unit root is rejected for all output series. Table 2 reports summary statistics for the growth rate of the industrial production index. We can see that $\mathrm{IE}_{t}$ exhibits the highest mean and standard deviation during both sample periods. Moreover, the Jarque-Bera statistics are statistically significant for several output series, indicating that the null hypothesis of normal distribution is rejected for these series.

\section{Empirical results}

This section uses the residual cross-correlation approach to investigate output correlation between European EMU member countries. The first stage involves the

Table 2. Summary statistics

\begin{tabular}{|c|c|c|c|c|c|c|c|c|c|c|}
\hline & $\mathrm{AT}_{t}$ & $\mathrm{BE}_{t}$ & $\mathrm{DE}_{t}$ & $\mathrm{ES}_{t}$ & $\mathrm{FI}_{t}$ & $\mathrm{FR}_{t}$ & $\mathrm{IE}_{t}$ & $\mathrm{IT}_{t}$ & $\mathrm{NL}_{t}$ & $\mathrm{PT}_{t}$ \\
\hline \multicolumn{11}{|c|}{ January 1994 to December 1998} \\
\hline$\mu$ & 0.39 & 0.16 & 0.18 & 0.38 & 0.52 & 0.23 & 1.24 & 0.19 & 0.09 & 0.39 \\
\hline$\sigma$ & 2.12 & 3.24 & 1.23 & 1.49 & 2.32 & 1.09 & 3.22 & 0.94 & 2.15 & 2.77 \\
\hline$m_{3}$ & 0.24 & 0.45 & -0.72 & 1.07 & 0.50 & 0.55 & 0.10 & 0.22 & -0.06 & 0.51 \\
\hline$m_{4}$ & 2.51 & 3.51 & 4.01 & 6.76 & 4.28 & 4.31 & 2.29 & 3.71 & 5.34 & 3.87 \\
\hline $\mathrm{JB}$ & 1.15 & 2.61 & $7.63 *$ & $45.99 *$ & $6.49 *$ & $7.22 *$ & 1.34 & 1.72 & $13.47 *$ & 4.38 \\
\hline
\end{tabular}

January 1999 to December 2003

\begin{tabular}{ccccccccccc}
$\mu$ & 0.34 & 0.18 & 0.14 & 0.11 & 0.31 & 0.10 & 0.87 & 0.07 & 0.07 & 0.09 \\
$\sigma$ & 2.10 & 2.52 & 1.19 & 1.44 & 2.93 & 0.81 & 6.58 & 0.75 & 1.86 & 3.10 \\
$m_{3}$ & 0.30 & -0.08 & -0.08 & 0.73 & 0.70 & 0.01 & 0.02 & 0.07 & -0.20 & 0.16 \\
$m_{4}$ & 4.63 & 3.49 & 2.56 & 5.81 & 8.14 & 2.55 & 3.45 & 2.66 & 4.82 & 5.62 \\
$\mathrm{JB}$ & $7.55^{*}$ & 0.67 & 0.54 & $25.00^{*}$ & $70.90^{*}$ & 0.51 & 0.51 & 0.35 & $8.65^{*}$ & $17.43^{*}$ \\
\hline
\end{tabular}

Table 2 reports mean $(\mu)$, standard deviation $(\sigma)$, skewness $\left(m_{3}\right)$, kurtosis $\left(m_{4}\right)$, and the Jarque-Bera statistic (JB) for each differenced series. *shows that the null hypothesis of normal distribution is rejected at the 5 percent significance level.

\footnotetext{
${ }^{3}$ We consider two regression equations $\mathrm{CT}$ and $\mathrm{C}$, where $\mathrm{CT}$ corresponds to the regression including a constant term and a liner trend, and $\mathrm{C}$ corresponds to the regression including a constant term only. In the augmented Dickey and Fuller (1979) test, the lag length of the augmentation term is selected by AIC. During the pre-EMU period, the lags for the $\mathrm{CT}$ and $\mathrm{C}$ are $(2,1)$ for $\mathrm{AT}_{t},(1,1)$ for $\mathrm{BE}_{t},(1,1)$ for $\mathrm{DE}_{t}$, $(0,1)$ for $\mathrm{ES}_{t},(1,1)$ for $\mathrm{FI}_{t},(0,0)$ for $\mathrm{FR}_{t},(3,3)$ for $\mathrm{IE}_{t},(1,1)$ for $\mathrm{IT}_{t},(1,1)$ for $\mathrm{NL}_{t}$, and $(1,1)$ for $\mathrm{PT}_{t}$, respectively. During the EMU period, the lags for the $\mathrm{CT}$ and $\mathrm{C}$ are $(3,1)$ for $\mathrm{AT}_{t},(3,3)$ for $\mathrm{BE}_{t},(0,0)$ for $\mathrm{DE}_{t},(1,1)$ for $\mathrm{ES}_{t},(1,1)$ for $\mathrm{FI}_{t},(0,0)$ for $\mathrm{FR}_{t},(1,1)$ for $\mathrm{IE}_{t},(0,0)$ for $\mathrm{IT}_{t},(2,2)$ for $\mathrm{NL}_{t}$, and $(5,5)$ for $\mathrm{PT}_{t}$, respectively. In the Phillips and Perron (1988) test, the truncation lag is set to 3.
} 
estimation of univariate time series models such as autoregressive (AR), moving average (MA), and ARMA models. Visual inspection suggests a little serial correlation for the growth rate of the industrial production index. To account for any possible weak serial correlation, we follow Hamori (2003) and specify $\operatorname{AR}(p)$ model:

$$
y_{t}=\phi_{0}+\sum_{j=1}^{p} \phi_{j} y_{t-j}+u_{t},
$$

where $y_{t}$ denotes the growth rate of the industrial production index. What has to be noticed is that the residual cross-correlation approach is based on the assumption of white noise residual series. Therefore, an important criterion for the choice of lag length is the absence of residual autocorrelation.

Table 3 and 4 present the ordinary least squares (OLS) estimates of univariate $\mathrm{AR}(p)$ models for $\mathrm{AT}_{t}, \mathrm{BE}_{t}, \mathrm{FI}_{t}, \mathrm{FR}_{t}, \mathrm{DE}_{t}, \mathrm{IE}_{t}, \mathrm{IT}_{t}, \mathrm{PT}_{t}, \mathrm{ES}_{t}$, and $\mathrm{NL}_{t}$. The lag length of the AR term is selected by Akaike information criterion (AIC). As seen in Table 3 and 4, most of AR parameter estimates are statistically significant at the 5 percent level, suggesting that almost all original series have the autocorrelation during both sample periods. Therefore, we see that the usual cross-correlation estimates between two original series are difficult to interpret because of the autocorrelation.

Table 3 and 4 also report diagnostic test statistics. LM is the Lagrange multiplier

Table 3. OLS estimates of univariate AR models: January 1994 to December 1998

\begin{tabular}{ccccccccccc}
\hline & $\mathrm{AT}_{t}$ & $\mathrm{BE}_{t}$ & $\mathrm{DE}_{t}$ & $\mathrm{ES}_{t}$ & $\mathrm{FI}_{t}$ & $\mathrm{FR}_{t}$ & $\mathrm{IE}_{t}$ & $\mathrm{IT}_{t}$ & $\mathrm{NL}_{t}$ & $\mathrm{PT}_{t}$ \\
\hline$\phi_{0}$ & $0.42^{*}$ & 0.18 & 0.17 & $0.37^{*}$ & $0.53^{*}$ & $0.23^{*}$ & $1.25^{*}$ & 0.15 & 0.16 & $0.37^{*}$ \\
& {$[0.09]$} & {$[0.19]$} & {$[0.09]$} & {$[0.14]$} & {$[0.14]$} & {$[0.09]$} & {$[0.17]$} & {$[0.22]$} & {$[0.09]$} & {$[0.13]$} \\
$\phi_{1}$ & $-0.80^{*}$ & $-0.69 *$ & $-0.47^{*}$ & $-0.32^{*}$ & $-0.61^{*}$ & $-0.46^{*}$ & $-0.47^{*}$ & 0.07 & $-0.76^{*}$ & $-0.75^{*}$ \\
& {$[0.14]$} & {$[0.12]$} & {$[0.13]$} & {$[0.12]$} & {$[0.13]$} & {$[0.12]$} & {$[0.13]$} & {$[0.13]$} & {$[0.14]$} & {$[0.12]$} \\
$\phi_{2}$ & $-0.54^{*}$ & -0.20 & -0.22 & & -0.21 & & -0.07 & $0.39^{*}$ & $-0.50^{*}$ & $-0.37^{*}$ \\
& {$[0.16]$} & {$[0.14]$} & {$[0.13]$} & & {$[0.13]$} & & {$[0.14]$} & {$[0.13]$} & {$[0.15]$} & {$[0.12]$} \\
$\phi_{3}$ & -0.23 & 0.20 & & & & & $-0.36^{*}$ & & -0.20 & \\
& {$[0.14]$} & {$[0.12]$} & & & & & {$[0.14]$} & & {$[0.13]$} & \\
$\phi_{4}$ & & & & & & & $-0.29^{*}$ & & & \\
& & & & & & & {$[0.13]$} & & & \\
$\mathrm{LM}$ & 10.84 & 10.55 & 8.36 & 8.48 & 13.46 & 19.94 & 7.71 & 9.87 & 9.60 & 12.22 \\
& $(0.54)$ & $(0.57)$ & $(0.76)$ & $(0.75)$ & $(0.34)$ & $(0.07)$ & $(0.81)$ & $(0.63)$ & $(0.65)$ & $(0.43)$ \\
\hline
\end{tabular}

Numbers in square parentheses are standard errors. Numbers in round parentheses are $p$-values. LM is the Lagrange multiplier test statistic for the null hypothesis that there is no residual autocorrelation up to order 12. The lag length is selected by AIC. *shows significance at the 5 percent level. 
Table 4. OLS estimates of univariate AR models: January 1999 to December 2003

\begin{tabular}{ccccccccccc}
\hline & $\mathrm{AT}_{t}$ & $\mathrm{BE}_{t}$ & $\mathrm{DE}_{t}$ & $\mathrm{ES}_{t}$ & $\mathrm{FI}_{t}$ & $\mathrm{FR}_{t}$ & $\mathrm{IE}_{t}$ & $\mathrm{IT}_{t}$ & $\mathrm{NL}_{t}$ & $\mathrm{PT}_{t}$ \\
\hline$\phi_{0}$ & $0.34^{*}$ & 0.18 & 0.13 & 0.10 & 0.30 & 0.11 & $0.81^{*}$ & 0.07 & 0.05 & 0.10 \\
& {$[0.13]$} & {$[0.14]$} & {$[0.11]$} & {$[0.08]$} & {$[0.21]$} & {$[0.08]$} & {$[0.37]$} & {$[0.08]$} & {$[0.10]$} & {$[0.08]$} \\
$\phi_{1}$ & $-0.43^{*}$ & $-0.38^{*}$ & $-0.34^{*}$ & $-0.59^{*}$ & $-0.46^{*}$ & $-0.30^{*}$ & $-0.66^{*}$ & -0.15 & $-0.48^{*}$ & $-0.84^{*}$ \\
& {$[0.12]$} & {$[0.13]$} & {$[0.12]$} & {$[0.12]$} & {$[0.13]$} & {$[0.12]$} & {$[0.13]$} & {$[0.12]$} & {$[0.13]$} & {$[0.13]$} \\
$\phi_{2}$ & $-0.39^{*}$ & $-0.33^{*}$ & & $-0.39^{*}$ & -0.20 & & $-0.26^{*}$ & & $-0.40^{*}$ & $-0.41^{*}$ \\
& {$[0.12]$} & {$[0.13]$} & & {$[0.12]$} & {$[0.13]$} & & {$[0.12]$} & & {$[0.13]$} & {$[0.16]$} \\
$\phi_{3}$ & & 0.17 & & & & & & & $-0.29^{*}$ & -0.31 \\
& & {$[0.14]$} & & & & & & & {$[0.12]$} & {$[0.16]$} \\
$\phi_{4}$ & & $-0.26^{*}$ & & & & & & & & $-0.45^{*}$ \\
& & {$[0.12]$} & & & & & & & & {$[0.16]$} \\
$\phi_{5}$ & & & & & & & & & & $-0.52^{*}$ \\
& & & & & & & & & & {$[0.16]$} \\
$\phi_{6}$ & & & & & & & & & & $-0.36^{*}$ \\
& & & & & & & & & & {$[0.13]$} \\
$\mathrm{LM}$ & 10.09 & 12.98 & 6.49 & 12.91 & 13.86 & 14.44 & 13.03 & 18.05 & 7.02 & 8.83 \\
& $(0.61)$ & $(0.37)$ & $(0.89)$ & $(0.38)$ & $(0.31)$ & $(0.27)$ & $(0.37)$ & $(0.11)$ & $(0.86)$ & $(0.72)$ \\
\hline
\end{tabular}

Numbers in square parentheses are standard errors. Numbers in round parentheses are $p$-values. LM is the Lagrange multiplier test statistic for the null hypothesis that there is no residual autocorrelation up to order 12 . The lag length is selected by AIC. *shows significance at the 5 percent level.

test statistic for the null hypothesis that there is no residual autocorrelation up to order 12. As Table 3 and 4 indicate, no rejections are found from the diagnostic tests for all models. Consequently, the selected models are empirically supported.

The second stage is to construct the sample cross-correlation function $\hat{\rho}_{u_{1} u_{2}}(k)$ between two residuals obtained from the univariate AR models. Computing the test statistic $\sqrt{T} \hat{\rho}_{u_{1} u_{2}}(k)$, we compare it with the critical value of $\mathrm{N}(0,1)$ at the 5 percent level. If $\sqrt{T} \hat{\rho}_{v_{1} v_{2}}(k)$ is larger than the critical value of $\mathrm{N}(0,1)$, then we reject the null hypothesis of no correlation between two time series. No correlation is equivalent to independence if the Guassian assumption applies to the joint distribution of the two time series. Therefore, the absence of output correlation (i.e., $\hat{\rho}_{u_{1} u_{2}}(k)=0$ for all $k$ ) implies that business cycles are independent for any two countries. From this viewpoint, we may say that the endogeneity of OCA hypothesis is empirically supported if significant pairs of countries for the EMU period increase compared with those for the pre-EMU period. Otherwise, the hypothesis is not empirically supported.

We shall now look at the contemporaneous residual cross-correlation function $\hat{\rho}_{u_{1} u_{2}}(0)$ for each sample period. Contemporaneous correlation, as Artis and Zhang 
(1999) suppose, can be regarded as the most important measure of business cycle synchronization, since the significance of contemporaneous correlation suggests that the economic shocks in a specific country spread instantaneously to another county and vice versa. In other words, contemporaneous correlation is directly related to output co-movement resulting in high business cycle synchronization. Results for the pre-EMU period are presented in Table 5, and results for the EMU period are presented in Table 6 . Table 5 reports that 11 pairs of countries are statistically significant at the 5 percent level. Table 6 , on the other hand, indicates that 23 pairs of countries are statistically significant at the 5 percent level. We can

Table 5. Contemporaneous residual cross-correlation $\hat{\rho}_{u_{1} u_{2}}(0)$ between EMU member countries: January 1994 to December 1998

\begin{tabular}{ccccccccccc}
\hline & $\mathrm{AT}_{t}$ & $\mathrm{BE}_{t}$ & $\mathrm{DE}_{t}$ & $\mathrm{ES}_{t}$ & $\mathrm{FI}_{t}$ & $\mathrm{FR}_{t}$ & $\mathrm{IE}_{t}$ & $\mathrm{IT}_{t}$ & $\mathrm{NL}_{t}$ & $\mathrm{PT}_{t}$ \\
\hline $\mathrm{AT}_{t}$ & 1.00 & & & & & & & & & \\
$\mathrm{BE}_{t}$ & 0.08 & 1.00 & & & & & & & & \\
$\mathrm{DE}_{t}$ & 0.07 & $-0.27^{*}$ & 1.00 & & & & & & & \\
$\mathrm{ES}_{t}$ & 0.09 & -0.05 & 0.21 & 1.00 & & & & & & \\
$\mathrm{FI}_{t}$ & $0.36^{*}$ & 0.04 & $0.32^{*}$ & -0.01 & 1.00 & & & & & \\
$\mathrm{FR}_{t}$ & $0.38^{*}$ & -0.09 & 0.11 & 0.14 & $0.34^{*}$ & 1.00 & & & & \\
$\mathrm{IE}_{t}$ & -0.09 & 0.04 & 0.06 & $0.34^{*}$ & 0.02 & $-0.31^{*}$ & 1.00 & & & \\
$\mathrm{IT}_{t}$ & 0.09 & -0.13 & 0.16 & $0.40^{*}$ & 0.16 & $0.31^{*}$ & 0.22 & 1.00 & & \\
$\mathrm{NL}_{t}$ & 0.17 & -0.05 & 0.08 & -0.03 & 0.23 & 0.16 & -0.05 & 0.25 & 1.00 & \\
$\mathrm{PT}_{t}$ & -0.06 & 0.01 & -0.05 & 0.10 & $0.28^{*}$ & -0.15 & $0.29 *$ & 0.22 & -0.19 & 1.00 \\
\hline
\end{tabular}

*shows significance at the 5 percent level.

Table 6. Contemporaneous residual cross-correlation $\hat{\rho}_{u_{1} u_{2}}(0)$ between EMU member countries: January 1999 to December 2003

\begin{tabular}{ccccccccccc}
\hline & $\mathrm{AT}_{t}$ & $\mathrm{BE}_{t}$ & $\mathrm{DE}_{t}$ & $\mathrm{ES}_{t}$ & $\mathrm{FI}_{t}$ & $\mathrm{FR}_{t}$ & $\mathrm{IE}_{t}$ & $\mathrm{IT}_{t}$ & $\mathrm{NL}_{t}$ & $\mathrm{PT}_{t}$ \\
\hline $\mathrm{AT}_{t}$ & 1.00 & & & & & & & & & \\
$\mathrm{BE}_{t}$ & $0.47^{*}$ & 1.00 & & & & & & & & \\
$\mathrm{DE}_{t}$ & $0.28^{*}$ & $0.27^{*}$ & 1.00 & & & & & & & \\
$\mathrm{ES}_{t}$ & $0.31^{*}$ & $0.47^{*}$ & $0.39^{*}$ & 1.00 & & & & & & \\
$\mathrm{FI}_{t}$ & 0.04 & $0.28^{*}$ & $0.32^{*}$ & 0.21 & 1.00 & & & & & \\
$\mathrm{FR}_{t}$ & $0.34^{*}$ & $0.37^{*}$ & $0.44^{*}$ & $0.37^{*}$ & $0.27^{*}$ & 1.00 & & & & \\
$\mathrm{IE}_{t}$ & 0.21 & 0.22 & $0.42^{*}$ & 0.08 & 0.08 & 0.02 & 1.00 & & & \\
$\mathrm{IT}_{t}$ & $0.39^{*}$ & $0.56^{*}$ & $0.33^{*}$ & $0.32^{*}$ & 0.10 & $0.33^{*}$ & $0.27^{*}$ & 1.00 & & \\
$\mathrm{NL}_{t}$ & 0.10 & 0.05 & 0.20 & 0.00 & 0.06 & $0.32^{*}$ & 0.18 & 0.13 & 1.00 & \\
$\mathrm{PT}_{t}$ & 0.06 & 0.24 & -0.05 & 0.09 & $0.34^{*}$ & $0.28^{*}$ & -0.04 & 0.04 & 0.14 & 1.00 \\
\hline
\end{tabular}

*shows significance at the 5 percent level. 
see from these empirical results that, after the transition to EMU, business cycles are more highly synchronized across EMU member countries.

In view of these empirical results, let us then examine the lagged residual crosscorrelation function $\hat{\rho}_{u_{1} u_{2}}(k)$ for each of the two sample period. Detection of lagged correlation is particularly important when contemporaneous correlation is not statistically significant, because it is possible that business cycles are dependent but there is no contemporaneous correlation. However, cross-dependence may not be consistent with business cycle synchronization. The significance of $\hat{\rho}_{u_{1} u_{2}}(k)$ implies that output series 2 affects output series 1 at lag $k$; business cycles are dependent but may not be synchronized when $\hat{\rho}_{u_{1} u_{2}}(k)$ with large absolute value of $k$ is significant. To put it another way, $\hat{\rho}_{u_{1} u_{2}}(k)$ should be significant for $k=0$ or small absolute value of $k$ when business cycles are synchronized between any two countries. Therefore, it seems reasonable to suppose that the endogeneity of OCA hypothesis is empirically supported if the residual cross-correlation estimates at shorter lags become significant during the EMU period.

Following Artis and Zhang (1995), this paper focuses on lagged crosscorrelation between Germany and another EMU member country. ${ }^{4}$ Let $y_{1 t}=\mathrm{DE}_{t}$, and $y_{2 t}=\mathrm{AT}_{t}, \mathrm{BE}_{t}, \mathrm{ES}_{t}, \mathrm{FI}_{t}, \mathrm{FR}_{t}, \mathrm{IE}_{t}, \mathrm{IT}_{t}, \mathrm{NL}_{t}$, or $\mathrm{PT}_{t}$. Table 7 reports the residual cross-correlation function for the pre-EMU period. We can see that contemporaneous correlation estimates hardly significant, but that most countries' business cycles are dependent on the German business cycle. In addition to this, it is also found that $\mathrm{AT}_{t}, \mathrm{BE}_{t}, \mathrm{FI}_{t}, \mathrm{IE}_{t}, \mathrm{IT}_{t}, \mathrm{NL}_{t}$, and $\mathrm{PT}_{t}$ have different lead-lag relationships to $\mathrm{DE}_{t}$, respectively. It must be noticed that these cross-correlation estimates are significant for different values of $k$. For example, there exists only contemporaneous correlation between $\mathrm{DE}_{t}$ and $\mathrm{FI}_{t}$, while there is evidence that $\mathrm{DE}_{t}$ and $\mathrm{IT}_{t}$ are independent. Thus, these empirical results make it clear that business cycles are dependent but not highly synchronized during the pre-EMU period.

Table 8 reports the residual cross-correlation function for the EMU period. As Table 8 indicates, it is clear that most countries' business cycles are also dependent on the German business cycle during the EMU period. Moreover, significant crosscorrelation estimates are obtained for $k=-3,0,3$, respectively (the only exception is $\mathrm{PT}_{t}$ ). Most of the significant cross-correlation estimates are obtained at lag 0 , and both nonzero lags, -3 and 3 , can be regarded as fully short lags in this analysis.

${ }^{4}$ To discus all pairs of countries is beyond the scope of this paper. When we test the significance of $\rho_{u_{1} u_{2}}(k)$ for $k= \pm 1, \ldots, \pm 12$, the number of correlation patterns exceeds 2,000 for both sample periods. 
Table 7. Lagged residual cross-correlation $\hat{\rho}_{u_{1} u_{2}}(0)$ between Germany and another EMU member country: January 1994 to December 1998

\begin{tabular}{|c|c|c|c|c|c|c|c|c|c|}
\hline $\mathrm{k}$ & $\mathrm{AT}_{t-k}$ & $\mathrm{BE}_{t-k}$ & $\mathrm{ES}_{t-k}$ & $\mathrm{FI}_{t-k}$ & $\mathrm{FR}_{t-k}$ & $\mathrm{IE}_{t-k}$ & $\mathrm{IT}_{t-k}$ & $\mathrm{NL}_{t-k}$ & $\mathrm{PT}_{t-k}$ \\
\hline-12 & 0.01 & -0.09 & 0.08 & -0.18 & 0.01 & 0.03 & -0.13 & -0.11 & 0.22 \\
\hline-11 & -0.12 & 0.02 & -0.05 & -0.21 & 0.09 & 0.01 & 0.02 & 0.00 & -0.13 \\
\hline-10 & -0.03 & 0.03 & 0.08 & -0.01 & -0.19 & $0.28 *$ & 0.02 & -0.02 & 0.21 \\
\hline-9 & 0.18 & -0.13 & -0.09 & 0.18 & 0.03 & -0.07 & -0.10 & -0.04 & -0.04 \\
\hline-8 & 0.16 & 0.01 & -0.05 & 0.23 & 0.20 & 0.21 & 0.18 & 0.09 & 0.00 \\
\hline-7 & 0.11 & 0.07 & 0.20 & -0.16 & -0.03 & 0.17 & -0.04 & -0.03 & -0.03 \\
\hline-6 & 0.14 & 0.03 & 0.10 & -0.10 & 0.05 & 0.01 & 0.11 & -0.05 & -0.14 \\
\hline-5 & -0.12 & 0.09 & -0.02 & 0.12 & -0.03 & 0.07 & 0.03 & -0.19 & 0.12 \\
\hline-4 & 0.20 & 0.09 & -0.03 & 0.09 & 0.05 & 0.17 & -0.09 & -0.02 & 0.04 \\
\hline-3 & 0.14 & 0.20 & 0.16 & 0.12 & 0.06 & -0.01 & 0.07 & 0.10 & 0.06 \\
\hline-2 & $0.47 *$ & 0.01 & 0.11 & 0.11 & 0.14 & 0.07 & 0.09 & 0.02 & -0.03 \\
\hline-1 & 0.21 & 0.00 & 0.25 & 0.18 & 0.20 & 0.03 & 0.19 & 0.05 & -0.13 \\
\hline 0 & 0.07 & $-0.27 *$ & 0.21 & $0.32 *$ & 0.11 & 0.07 & 0.16 & 0.08 & -0.05 \\
\hline 1 & -0.01 & 0.13 & -0.09 & 0.02 & -0.04 & -0.04 & -0.02 & 0.00 & 0.03 \\
\hline 2 & 0.12 & -0.01 & -0.15 & 0.06 & 0.18 & -0.14 & -0.11 & $0.30 *$ & -0.20 \\
\hline 3 & 0.10 & 0.11 & $0.35 *$ & 0.06 & $0.36^{*}$ & -0.21 & 0.10 & -0.13 & 0.10 \\
\hline 4 & -0.01 & 0.08 & 0.17 & -0.14 & -0.08 & 0.18 & -0.01 & -0.11 & -0.08 \\
\hline 5 & 0.03 & $-0.36^{*}$ & -0.05 & 0.17 & -0.01 & 0.11 & 0.11 & -0.05 & 0.11 \\
\hline 6 & -0.02 & 0.00 & 0.03 & 0.03 & 0.19 & -0.09 & -0.02 & $0.31 *$ & $-0.44 *$ \\
\hline 7 & 0.06 & -0.06 & -0.18 & 0.01 & 0.20 & $-0.26^{*}$ & -0.05 & 0.05 & -0.15 \\
\hline 8 & $-0.35^{*}$ & 0.09 & 0.13 & -0.03 & -0.11 & 0.11 & 0.17 & 0.11 & 0.05 \\
\hline 9 & 0.02 & -0.06 & 0.21 & 0.00 & -0.07 & 0.03 & 0.19 & 0.08 & -0.02 \\
\hline 10 & -0.01 & 0.05 & -0.15 & -0.08 & 0.06 & -0.13 & -0.08 & -0.20 & -0.05 \\
\hline 11 & -0.03 & -0.10 & -0.19 & -0.14 & -0.05 & -0.01 & -0.15 & -0.02 & 0.03 \\
\hline 12 & -0.23 & 0.06 & 0.12 & -0.12 & -0.09 & -0.01 & 0.10 & -0.17 & -0.01 \\
\hline
\end{tabular}

$\mathrm{DE}_{t}$ lags another if $\hat{\rho}_{u_{1} u_{2}}(k) \neq 0$ for some $k>0 ; \mathrm{DE}_{t}$ leads another if $\hat{\rho}_{u_{1} u_{2}}(k) \neq 0$ for some $k<0$. *shows significance at the 5 percent level.

These empirical results show clearly that cross-correlation estimates at shorter lags become significant. Therefore, it follows from the empirical results that disparities in lead-lag relationships reported in Table 7 disappear considerably during the EMU period. Judging from correlation patterns with Germany, we see that the degree of business cycle synchronization increases after the transition to EMU. On these grounds, it seems reasonable to conclude that the endogeneity of OCA hypothesis is empirically supported.

Our analysis makes it possible to interpret the future course of the European EMU. Since 1 January 1999, each European EMU member country has adopted 
Table 8. Lagged residual cross-correlation $\hat{\rho}_{u_{1} u_{2}}(k)$ between Germany and another EMU member country: January 1999 to December 2003

\begin{tabular}{|c|c|c|c|c|c|c|c|c|c|}
\hline $\mathrm{k}$ & $\mathrm{AT}_{t-k}$ & $\mathrm{BE}_{t-k}$ & $\mathrm{ES}_{t-k}$ & $\mathrm{FI}_{t-k}$ & $\mathrm{FR}_{t-k}$ & $\mathrm{IE}_{t-k}$ & $\mathrm{IT}_{t-k}$ & $\mathrm{NL}_{t-k}$ & $\mathrm{PT}_{t-k}$ \\
\hline-12 & 0.03 & -0.17 & -0.09 & -0.13 & 0.00 & 0.01 & 0.00 & 0.03 & -0.06 \\
\hline-11 & -0.05 & -0.12 & -0.19 & -0.16 & 0.04 & 0.10 & -0.13 & -0.09 & 0.12 \\
\hline-10 & 0.11 & 0.00 & -0.20 & 0.11 & 0.10 & -0.12 & 0.02 & -0.04 & 0.18 \\
\hline-9 & 0.02 & -0.16 & -0.12 & -0.15 & -0.25 & 0.11 & -0.07 & -0.04 & -0.21 \\
\hline-8 & -0.07 & -0.01 & 0.13 & 0.07 & 0.12 & -0.20 & 0.00 & 0.04 & 0.01 \\
\hline-7 & 0.15 & 0.09 & 0.17 & 0.00 & 0.18 & 0.18 & -0.05 & -0.01 & 0.05 \\
\hline-6 & -0.04 & -0.04 & -0.01 & 0.03 & 0.09 & 0.02 & 0.01 & 0.25 & 0.08 \\
\hline-5 & 0.07 & 0.07 & -0.05 & 0.15 & 0.17 & -0.14 & 0.10 & -0.03 & -0.01 \\
\hline-4 & 0.10 & 0.09 & 0.10 & -0.13 & -0.18 & -0.01 & 0.10 & -0.14 & -0.01 \\
\hline-3 & 0.06 & -0.11 & 0.02 & 0.09 & 0.11 & 0.04 & 0.12 & $0.34^{*}$ & 0.18 \\
\hline-2 & 0.06 & 0.06 & 0.04 & 0.11 & 0.02 & -0.01 & -0.15 & -0.14 & -0.03 \\
\hline-1 & 0.11 & 0.08 & 0.01 & 0.19 & -0.01 & 0.15 & 0.22 & 0.02 & 0.12 \\
\hline 0 & $0.28 *$ & $0.27 *$ & $0.39 *$ & $0.32 *$ & $0.44 *$ & $0.42 *$ & $0.33 *$ & 0.20 & -0.05 \\
\hline 1 & 0.12 & 0.00 & 0.20 & -0.21 & 0.19 & -0.04 & 0.22 & 0.23 & -0.15 \\
\hline 2 & 0.09 & 0.02 & -0.09 & 0.04 & 0.03 & -0.04 & 0.04 & 0.22 & 0.10 \\
\hline 3 & -0.03 & 0.21 & 0.14 & $0.28 *$ & 0.11 & 0.11 & 0.24 & 0.04 & -0.06 \\
\hline 4 & 0.01 & -0.02 & -0.07 & 0.13 & 0.03 & -0.06 & 0.21 & 0.04 & -0.09 \\
\hline 5 & 0.13 & 0.13 & 0.13 & 0.22 & 0.03 & 0.11 & -0.16 & 0.15 & 0.10 \\
\hline 6 & 0.22 & 0.17 & 0.12 & -0.19 & 0.01 & 0.09 & 0.22 & -0.17 & -0.01 \\
\hline 7 & -0.12 & -0.06 & 0.09 & 0.22 & 0.12 & -0.07 & -0.16 & -0.10 & -0.12 \\
\hline 8 & 0.05 & 0.07 & 0.10 & 0.09 & -0.01 & 0.06 & 0.15 & 0.01 & -0.08 \\
\hline 9 & 0.20 & 0.15 & 0.11 & -0.14 & -0.12 & -0.03 & 0.15 & 0.12 & 0.10 \\
\hline 10 & 0.06 & -0.13 & 0.14 & -0.03 & 0.17 & 0.03 & -0.01 & 0.02 & -0.05 \\
\hline 11 & -0.18 & -0.18 & -0.25 & -0.13 & 0.12 & -0.25 & -0.12 & -0.05 & -0.19 \\
\hline 12 & -0.10 & -0.12 & 0.10 & 0.08 & -0.11 & -0.19 & -0.20 & -0.13 & 0.14 \\
\hline
\end{tabular}

$\mathrm{DE}_{t}$ lags another if $\hat{\rho}_{u_{1} u_{2}}(k) \neq 0$ for some $k<0 ; \mathrm{DE}_{t}$ leads another if $\hat{\rho}_{u_{1} u_{2}}(k) \neq 0$ for some $k<0 . *$ shows significance at the 5 percent level.

the euro as a common currency and, consequently, lost the ability to conduct a national monetary policy. The degree of business cycle synchronization is a very important matter in this respect, because a country with idiosyncratic business cycle cannot conduct an independent counter-cyclic monetary policy. The important point to note is that inevitable costs of joining EMU depend largely on the degree of business cycle synchronization across EMU member countries. It is clear that the endogeneity of OCA is relevant to the costs consequent on the participation in EMU, and thus we can see from our empirical results that the costs have a tendency to decrease. The European EMU is likely to succeed as long as the endogeneity of OCA holds in the case of the euro area, ceteris paribus. Our 
empirical results support the European EMU in this sense. ${ }^{5}$

\section{Conclusion}

This paper is intended as an empirical investigation on the endogenous nature of business cycle synchronization. From the theoretical point of view, there are two directly-opposed ideas concerning the impact of EMU on the degree of business cycle synchronization across EMU member countries. One possibility is to assume that EMU leads to an increase in the degree of business cycle synchronization. Another possibility is that EMU gives rise to a decrease in the degree of business cycle synchronization. Consequently, this issue has been studied empirically by many researchers.

Many previous studies provide empirical evidence to suggest that economic integration tends to increase the degree of business cycle synchronization across countries. What seems to be lacking, however, is the analysis on the timing of correlation. To examine correlation patterns in detail, this paper uses the residual cross-correlation approach. Unlike the usual cross-correlation function, the residual cross-correlation function is much easier to handle and interpret because it filters out the autocorrelation of each original series.

Using output data for European EMU member countries, we obtain the following empirical results: (i) contemporaneous correlation estimates become more significant during the EMU period; (ii) business cycles are dependent but not highly synchronized during the pre-EMU period; (iii) after the transition to EMU, disparities in lead-lag relationships for the pre-EMU period disappear considerably and thus business cycles are more highly synchronized than before. On these grounds, we conclude that the endogeneity of OCA hypothesis holds in the case of the euro area. It may be that the euro area turns into the OCA endogenously.

Although our empirical results imply that inevitable costs of joining EMU have a tendency to decrease, we cannot say from the results that high business cycle synchronization is fully realized in the entire euro area economy. For example, significant contemporaneous residual cross-correlation estimates for the EMU period are obtained from 23 pairs of countries, but remaining 22 pairs of countries are not significant. This finding is also confirmed in Table 9 and 10. Table 9 and 10

\footnotetext{
${ }^{5}$ As shown in Table 6 and 8, perfect business cycle synchronization is not realized in the euro area. Therefore, our empirical analysis suggests that inevitable costs of joining EMU have a tendency to decrease in the euro area, but that much cost still remains to be born among European EMU member countries.
} 
present the usual contemporaneous cross-correlation function $\hat{\rho}_{y_{1} y_{2}}(0)$ between original output series. Values of contemporaneous correlation estimates have a tendency to rise, while these values vary according to pairs of countries.

It must be noted that much cost still remains to be born among European EMU member countries in the present circumstances. One thing, however, is certain: the European EMU leads to an increase in the degree of business cycle synchronization across member countries, and thus costs arising from asymmetric business cycles tend to decrease. The costs will further decrease in the long run. An implication of this paper is that the European EMU is likely to succeed as long as the degree of

Table 9. Contemporaneous cross-correlation $\hat{\rho}_{y_{1} y_{2}}(0)$ between EMU member countries: January 1994 to December 1998

\begin{tabular}{ccccccccccc}
\hline & $\mathrm{AT}_{t}$ & $\mathrm{BE}_{t}$ & $\mathrm{DE}_{t}$ & $\mathrm{ES}_{t}$ & $\mathrm{FI}_{t}$ & $\mathrm{FR}_{t}$ & $\mathrm{IE}_{t}$ & $\mathrm{IT}_{t}$ & $\mathrm{NL}_{t}$ & $\mathrm{PT}_{t}$ \\
\hline $\mathrm{AT}_{t}$ & 1.00 & & & & & & & & & \\
$\mathrm{BE}_{t}$ & 0.09 & 1.00 & & & & & & & & \\
$\mathrm{DE}_{t}$ & 0.00 & -0.39 & 1.00 & & & & & & & \\
$\mathrm{ES}_{t}$ & -0.07 & -0.09 & 0.17 & 1.00 & & & & & & \\
$\mathrm{FI}_{t}$ & 0.33 & -0.10 & 0.22 & -0.06 & 1.00 & & & & & \\
$\mathrm{FR}_{t}$ & 0.39 & 0.00 & 0.02 & 0.05 & 0.30 & 1.00 & & & & \\
$\mathrm{IE}_{t}$ & -0.14 & -0.11 & 0.11 & 0.43 & -0.03 & -0.39 & 1.00 & & & \\
$\mathrm{IT}_{t}$ & 0.08 & 0.00 & 0.04 & 0.21 & 0.30 & 0.47 & -0.06 & 1.00 & & \\
$\mathrm{NL}_{t}$ & 0.26 & -0.04 & 0.00 & -0.04 & 0.33 & 0.17 & 0.06 & 0.32 & 1.00 & \\
$\mathrm{PT}_{t}$ & 0.04 & -0.18 & 0.02 & 0.11 & 0.35 & -0.07 & 0.21 & 0.20 & -0.15 & 1.00 \\
\hline
\end{tabular}

Table 9 presents the usual contemporaneous cross-correlation function $\hat{\rho}_{y_{1} y_{2}}(0)$ between original output series.

Table 10. Contemporaneous cross-correlation $\hat{\rho}_{y_{1} y_{2}}(0)$ between EMU member countries: January 1999 to December 2003

\begin{tabular}{lcccccccccc}
\hline & $\mathrm{AT}_{t}$ & $\mathrm{BE}_{t}$ & $\mathrm{DE}_{t}$ & $\mathrm{ES}_{t}$ & $\mathrm{FI}_{t}$ & $\mathrm{FR}_{t}$ & $\mathrm{IE}_{t}$ & $\mathrm{IT}_{t}$ & $\mathrm{NL}_{t}$ & $\mathrm{PT}_{t}$ \\
\hline $\mathrm{AT}_{t}$ & 1.00 & & & & & & & & & \\
$\mathrm{BE}_{t}$ & 0.49 & 1.00 & & & & & & & & \\
$\mathrm{DE}_{t}$ & 0.14 & 0.30 & 1.00 & & & & & & & \\
$\mathrm{ES}_{t}$ & 0.35 & 0.52 & 0.34 & 1.00 & & & & & & \\
$\mathrm{FI}_{t}$ & -0.08 & 0.25 & 0.36 & 0.20 & 1.00 & & & & & \\
$\mathrm{FR}_{t}$ & 0.30 & 0.57 & 0.35 & 0.38 & 0.24 & 1.00 & & & & \\
$\mathrm{IE}_{t}$ & -0.04 & 0.07 & 0.41 & 0.07 & 0.00 & -0.08 & 1.00 & & & \\
$\mathrm{IT}_{t}$ & 0.34 & 0.35 & 0.23 & 0.22 & 0.09 & 0.19 & 0.20 & 1.00 & & \\
$\mathrm{NL}_{t}$ & 0.12 & 0.23 & 0.05 & 0.00 & 0.10 & 0.25 & 0.21 & 0.07 & 1.00 & \\
$\mathrm{PT}_{t}$ & 0.14 & 0.36 & 0.03 & 0.24 & 0.45 & 0.33 & -0.21 & 0.13 & 0.20 & 1.00 \\
\hline
\end{tabular}

Table 10 presents the usual contemporaneous cross-correlation function $\hat{\rho}_{y_{1} y_{2}}(0)$ between original output series. 
business cycle synchronization across member countries has a tendency to increase in the euro area. Our empirical results support the European EMU in this respect.

\section{Acknowledgements}

The author would like to thank Seiichi Fujita and Shigeyuki Hamori. Of course I am solely responsible for any errors.

Received 4 August 2005, Accepted 29 December 2005

\section{References}

Artis, M., and W. Zhang (1995) International Business Cycles and the ERM: Is There a European Business Cycle?, International Journal of Finance and Economics, 2, 1-16 Artis, M., and W. Zhang (1999) Further Evidence on the International Business Cycle and the ERM: Is there a European Business Cycle?, Oxford Economic Papers, 51, 120-132

De Grauwe, P. (2005) Endogeneities of Optimum Currency Areas: What Brings Countries Sharing a Single Currency Closer Together?, ECB Working Paper, No.468

Frankel, J., and A. Rose (1998) The Endogeneity of the Optimum Currency Area Criteria, Economic Journal, 108, 1009-1025

Frankel, J., and A. Rose (2002) An Estimate of the Effect of Common Currencies on Trade and Income, Quarterly Journal of Economics, 117, 437-466

Kalemli-Ozcan, S., B.E. Sørensen, and O. Yosha (2003) Risk Sharing and Industrial Specialization: Regional and International Evidence, American Economic Review, 93, 903-918

Krugman, P. (1991) Geography and Trade, Cambridge, MA: MIT Press

Hamori, S. (2003) An Empirical Investigation of Stock Markets: The CCF Approach, Kluwer Academic Publishers

Haugh, L.D. (1976) Checking the Independence of Two Covariance-stationary Time Series: A Univariate Residual Cross-correlation Approach, Journal of the American Statistical Association, 71, 378-385

Mélitz, J. (2004) Risk Sharing and EMU, Journal of Common Market Studies, 42, 815-840

Ramos, R., M. Clar, and J. Suriñach (2003) National Versus Sectoral Shocks: New Evidence for the Manufacturing Sector in European Countries, Economics Letters, $78,241-245$

Rose, A., and C. Engle (2002) Currency Union and International Integration, Journal of Money, Credit, and Banking, 34, 1067-1089 